\title{
Comparação entre mapas de solos obtidos por sensoriamento remoto espectral e pelo método convencional
}

\author{
José Alexandre M. Demattê( ${ }^{(1)}$, Aline Marques Genú( ${ }^{(1)}$, Peterson Ricardo Fiorio(2), Jonas Luís Ortiz ${ }^{(3)}$, \\ Jairo Antônio Mazza ${ }^{(1)}$ e Hudson Carlos Lissoni Leonardo(3)
}

\begin{abstract}
(1)Universidade de São Paulo (USP), Escola Superior de Agricultura "Luiz de Queiroz" (Esalq), Dep. de Solos e Nutrição de Plantas, Caixa Postal 9, CEP 13418-900 Piracicaba, SP. E-mail:jamdemat@esalq.usp.br, amgenu@esalq.usp.br, jamazza@esalq.usp.br (2)Universidade Estadual do Oeste do Paraná, Dep. de Ciências Agrárias, Rua Pernambuco, 1777, Caixa Postal 91, CEP 85960-000 Marechal Cândido Rondon, PR. E-mail: fiorio@unioeste.br (3)USP-Esalq, Dep. de Ciências Florestais. E-mail:jlortiz@esalq.usp.br, hclleona@esalq.usp.br
\end{abstract}

\begin{abstract}
Resumo - O meio científico carece de métodos que efetivem a utilização da espectroradiometria em mapeamentos de solos. O objetivo deste trabalho foi comparar o uso do sensoriamento remoto espectral com o método convencional, na obtenção de mapas de solos. Utilizando o mapa planialtimétrico como base, trinta pontos de amostragem foram demarcados na área de estudo. As amostras foram coletadas a 0-20, 40-60 e 80-100 cm de profundidade em cada ponto, todos georreferenciados, totalizando noventa amostras nas quais foram feitas as análises químicas, físicas e radiométricas. Os dados radiométricos foram obtidos em laboratório usando o espectrorradiômetro IRIS, na faixa de 400-2.500 nm. Pela análise e interpretação dos dados espectrais juntamente com as curvas de nível, obteve-se um mapa espectral detalhado de solos. Na mesma área foi obtido um mapa de solos detalhado pelo método convencional. Foram identificadas quatro classes de solos em ambos os mapas, com boa correlação, demonstrando que os dados espectrais podem ser utilizados como instrumento no mapeamento de solos. A utilização conjunta dos dados de sensoriamento remoto espectral, curvas de nível e trabalho de campo permite a obtenção do mapa detalhado de solos.
\end{abstract}

Termos para indexação: mapeamento de solos, radiometria, geoprocessamento.

\section{Comparison between soil maps obtained by spectral remote sensing and conventional method}

\begin{abstract}
The objective of this work was to compare the use of the spectral remote sensing for producing soil maps to the conventional method. Using a planialtimetric base map, thirty sampling points were demarcated in the studied area. The samples were collected at 0-20, 40-60 and 80-100 cm depth in each point, all georreferenced, with the total of ninety samples in which the chemical, physical and radiometric analyses were performed. The radiometric data were obtained in laboratory using the spectroradiometer IRIS, in the range of 400-2,500 nm. Using the analyses and interpretation of both the spectral and the planialtimetric data, a detailed spectral soil map was obtained. For the same area, a conventional detailed map was also obtained. Four soil classes were identified in both maps. The produced maps presented good correlation, demonstrating that spectral data can be used as tool in soil survey. The use of spectral remote sensing, planialtimetric data and field work allows the obtention of a detailed soil map.
\end{abstract}

Index terms: soil survey, radiometry, geoprocessing.

\section{Introdução}

A distribuição espacial das diferentes classes de solos que ocorrem em uma área é normalmente visualizada a partir de mapas pedológicos. No Brasil, os levantamentos são antigos e pouco detalhados, como os do projeto Radam (escala 1:1.000.000) e os realizados pela Embrapa. O Estado de São Paulo é o que apresenta mapas com melhor nível de detalhamento, tais como as cartas semi-detalhadas feitas pelo IAC que, mesmo assim, não cobrem todo o Estado.

Com a crescente preocupação ambiental, existe uma necessidade do monitoramento das condições dos recursos naturais e da implantação da agricultura de precisão. As informações sobre os solos devem ser conciliáveis com essa realidade. Entretanto, para que isso seja possível, é preciso que novos mapas sejam fei- 
tos e em escala compatível com o objetivo. Porém, o método tradicional é lento, caro e requer análises laboratoriais subsequientes (McBratney et al., 2003). Desta forma, novos métodos que tornem os levantamentos de solos mais rápidos e menos onerosos são necessários.

Uma das técnicas que podem auxiliar neste aspecto é o sensoriamento remoto em todos os níveis de aquisição de dados, como o orbital, utilizando-se sensores multiespectrais (TM e ETM do Landsat), hiperespectrais (Aster) e de alta resolução espacial (Ikonos); aéreos (fotos aéreas) e videografia. Informações obtidas por sensores terrestres e em laboratório também são utilizadas (Coleman et al., 1993; Demattê et al., 2001; Fiorio, 2002).

A aplicação desta técnica no estudo do solo baseiase no fato de que os diferentes solos absorvem e refletem a energia eletromagnética em comprimentos de onda distintos, de acordo com seus atributos químicos, físicos e mineralógicos sendo, desta forma, possível diferenciálos entre si (Andronikov \& Dobrovolskiy, 1991).

Assim, Demattê \& Garcia (1999), estudando solos derivados de rochas básicas do Estado do Paraná, discriminaram o Brunizem Avermelhado, a Terra Roxa Estruturada e o Latossolo Roxo a partir de suas curvas espectrais. Demattê et al. (2001) verificaram estreita correlação entre as unidades de solos discriminadas por radiometria e mapeamento convencional, atestando sua utilidade como instrumento auxiliar no mapeamento de solos.

A avaliação espectral de solos pelo sensoriamento remoto também permite a quantificação de atributos do solo e para isto, diversos métodos têm sido testados. Ben-Dor \& Banin (1990) determinaram correlações significativas entre reflectância e os teores de $\mathrm{Al}_{2} \mathrm{O}_{3}, \mathrm{MgO}$ e $\mathrm{Fe}_{2} \mathrm{O}_{3}$, enquanto Coleman et al. (1991) desenvolveram equações para estimar o conteúdo de matéria orgânica, argila e óxidos de ferro a partir de dados espectrais para oito solos do Alabama, Estados Unidos.

Recentemente, Ben-Dor (2002) constatou a importância desse instrumento no estudo das características químicas e mineralógicas dos solos, assim como na separação de unidades de solos usando sensores em laboratório e campo. Portanto, o conhecimento da resposta espectral das diferentes classes de solos torna-se fundamental na distinção e separação das unidades de solos existentes em determinada região. A questão ainda não explorada é como trabalhar com estas informações visando gerar um mapa de solos.
O objetivo deste trabalho foi comparar o uso do sensoriamento remoto espectral com o método convencional na obtenção de mapas de solos.

\section{Material e Métodos}

A área de estudo, com 1.300 ha, localiza-se na região do Município de Uberlândia, MG, e tem sido destinada ao cultivo de soja. De acordo com a classificação de Köppen, o clima é do tipo Aw, caracterizado por uma estação chuvosa nos períodos de outubro a novembro e de março a abril, e por um período seco entre maio e setembro. O relevo é plano a suave ondulado e, nas áreas de baixada próximo aos córregos, o solo apresenta sinais de encharcamento.

Na obtenção dos mapas de solos, os procedimentos abaixo descritos foram realizados.

\section{Fase 1: coleta de dados}

Com o auxílio do mapa planialtimétrico, na escala de 1:10.000, e utilizando as características do relevo como apoio, a área foi percorrida em diversas secções, sempre no sentido da cota mais baixa para a mais alta. Foram estabelecidos 30 pontos de coleta de amostras de solo, todos georreferenciados. Cada ponto foi amostrado em três profundidades (0-20, 40-60 e 80-100 cm), designadas, respectivamente, camadas $\mathrm{A}, \mathrm{B}$ e $\mathrm{C}$, totalizando 90 amostras. As amostras coletadas foram moídas, peneiradas em malha $2 \mathrm{~mm}$ e secadas em estufa a $45^{\circ} \mathrm{C}$ por 24 horas.

\section{Fase 2: análises de solo}

A análise granulométrica foi realizada pelo método do densímetro, usando o hexametafosfato de sódio como dispersante (Camargo et al., 1986). A análise química constou da determinação do $\mathrm{pH}$ em $\mathrm{CaCl}_{2}, \mathrm{H}_{2} \mathrm{O}$ e $\mathrm{KCl}$, teor de matéria orgânica (MO), $\mathrm{P}$ (resina), $\mathrm{K}, \mathrm{Ca}, \mathrm{Mg}$, Al, CTC, saturação por bases e saturação por alumínio, conforme Raij et al. (1987).

\section{Fase 3: aquisição de dados espectrais}

Os dados espectrais de cada amostra foram obtidos por meio do sensor Infra Red Intelligent Spectroradiometer (IRIS) (GER, 1996). A faixa espectral coberta por esse espectrorradiômetro estende-se de 400 a $2.500 \mathrm{~nm}$, com uma resolução espectral de $2 \mathrm{~nm}$ entre 400 e $1.000 \mathrm{~nm}$ e de $4 \mathrm{~nm}$ entre 1.000 e $2.500 \mathrm{~nm}$. A geometria do sistema foi baseada no posicionamento perpendicular do sensor em relação à amostra, que estava acondicionada em placa de Petri, mantendo entre ambos uma distância de $27 \mathrm{~cm}$. 
A fonte de iluminação foi posicionada a $61 \mathrm{~cm}$ da amostra, formando um ângulo de $20^{\circ}$ com o zênite. O padrão de referência absoluto utilizado foi uma placa de espectralon branca. A razão espectral entre o fluxo de radiação refletido pela superfície de referência e a amostra gerou o fator de reflectância bidirecional (Nicodemus et al., 1977).

\section{Fase 4: interpretação dos dados espectrais}

Os dados espectrais foram analisados e interpretados para identificar e classificar áreas de solos distintos. Foram utilizados três métodos. No primeiro deles, para cada ponto de tradagem foram obtidas três amostras de terra e curvas espectrais respectivas a cada profundidade. Todas as curvas foram avaliadas e agrupadas quanto à forma, intensidade e bandas de absorção de acordo com os padrões de curvas de solos registrados por Epiphanio et al. (1992), Formaggio et al. (1996) e Demattê et al. (2001). No segundo método, as curvas foram analisadas pelos resultados de sua derivada segunda, conforme Savitzky \& Golay (1964), auxiliando na identificação dos óxidos de ferro (hematita e goethita), caulinita, gibbsita, enquanto no terceiro método foi realizada uma análise quantitativa baseada na divisão do espectro analisado (450-2.500 nm) em 22 bandas e 13 alturas.

A curva espectral apresenta várias informações ao longo do espectro. O número de bandas determinadas para o sensor utilizado é de 825 e a interpretação do significado de cada banda e sua relação com o solo é reconhecidamente uma tarefa complexa. Para suplantar este problema, o espectro que compreende o intervalo de 450 a $2.500 \mathrm{~nm}$ é dividido, escolhendo-se as bandas mais significativas. A escolha das bandas baseia-se em observações empíricas e, simultaneamente, na literatura. $\mathrm{O}$ objetivo é determinar um menor número de bandas que possam ser modelados por sistemas estatísticos e que, por sua vez, representem o referido solo. Portanto, considerando as bandas de absorção de elementos do solo já descritos e bem caracterizados como os óxidos de ferro (481 nm), água e grupos $\mathrm{OH}$ $(1.417$ e $1.927 \mathrm{~nm})$, caulinita $(2.206 \mathrm{~nm})$ e gibbsita $(2.258 \mathrm{~nm})$, e em observações de curvas espectrais de vários solos, Nanni (2000) indicou a existência de 22 bandas-chave. No modelo é inserida a magnitude de reflectância de cada solo (cada banda refere-se a um comprimento de onda específico).

Com base no método de Nanni (2000), também foram determinadas as alturas, em um total de treze.
A altura é caracterizada pela diferença da reflectância entre dois comprimentos de onda que determinam uma absorção de energia. Desta forma, tem-se as bandas (nm) B1 (450-481), B2 (481), B3 (481-596), B4 (596-710), B5 (710-814), B6 (814-975), B7 (975-1350), B8 (1350-1417), B9 (1417), B10 (1417-1449), B11 (1449-1793), B12 (1793-1831), B13 (1865-1927), B14 (1927), B15 (1927-2101), B 16 (2101-2139),B17 (2139-2206), B18 (2206), B19 (2206-2258), B20 (2258), B21(2258-2389) e B22 (2389-2498) e alturas (nm) H1 (469-532), H2 (536-768), H3 (768-876), H4 (876-1.353), H5 (1.353-1.411), H6 (1.411-1.439), H7 (1.439-1.783), H8 (1.860-1.923), H9 (1.923-2.120), H10 (2.120-2.206), H11 (2.206-2.258), H12 (2.258-2.389), H13 (2.389-2.498).

Fase 5: determinação do mapa de solos espectral Nesta fase o objetivo foi utilizar os dados espectrais das curvas das amostras de terra para agrupar solos semelhantes e auxiliar na delimitação das unidades de mapeamento, juntamente com as curvas de nível.

Primeiramente, foram impressas as curvas espectrais das amostras de terra de cada local de tradagem para, então, realizar a interpretação descritiva de cada uma nas três profundidades de amostragem, simultaneamente. O processo é semelhante à interpretação de análise de solo de cada local de tradagem, porém, nesta fase não foram observadas as análises mas apenas as informações espectrais.

O processo de interpretação, conforme descrito na fase 4 , permitiu agrupar em curvas espectrais de tradagens semelhantes. Como apoio quantitativo ao processo interpretativo, foi realizada uma análise estatística de agrupamentos (Cluster Analysis) desenvolvida no programa Statistical Analysis System (SAS Institute, 1999). Esta análise utilizou-se das informações espectrais de cada tradagem nas três profundidades simultaneamente. O sistema comparou todas as 30 tradagens, 90 curvas espectrais, determinando os grupos de curvas mais semelhantes. As informações descritivas e as qualitativas auxiliaram na decisão sobre grupos de solos definitivos. Considerou-se, nesta fase, que cada grupo gerado pertence a determinado solo. Com base nos grupos semelhantes e simultaneamente nas informações de curvas de nível, foi realizada a delimitação das unidades de mapeamento.

Finalmente, e seguindo os mesmos passos do método tradicional de levantamento, foram demarcados perfis nas áreas mais homogêneas de cada grupo de solo. Nos perfis foi realizada a descrição morfológica (Le- 
mos \& Santos, 1996) e coleta de amostra de terra de cada horizonte. Essas amostras foram encaminhadas para análise espectral. As análises químicas e granulométricas também foram realizadas, porém estes dados foram interpretados na fase de determinação do mapeamento tradicional.

Com base nas curvas espectrais dos perfis, foi realizada a interpretação comparativa com padrões previamente descritos. Os padrões utilizados foram os de Epiphanio et al. (1992), Formaggio et al. (1996) e Demattê et al. (2001). Esses autores correlacionaram curvas espectrais de perfis de solos e a respectiva classificação. Comparando as curvas dos perfis de solo do presente trabalho com as destes autores, foi possível chegar a uma classificação até o segundo nível categórico (subordem). Com isso, determinou-se o mapa detalhado de solos, considerando-se informações espectrais e curvas de nível.

Fase 6: desenvolvimento do mapa de solos detalhado pelo método convencional

O mapa convencional de solos foi obtido analisandose os dados químicos e granulométricos das 90 amostras de terra. Em seguida, observando-se as curvas de nível, quatro unidades de mapeamento foram delimitadas e classificadas após a descrição morfológica (Lemos \& Santos, 1996) de acordo com Embrapa (1999). Os mapas foram digitalizados e introduzidos no sistema de informação geográfica Spring (Instituto Nacional de Pesquisas Espaciais, 1999).

\section{Fase 7: validação do mapa espectral}

O mapa de solos convencional e o espectral foram comparados por meio do cruzamento de suas informações, pelo emprego de técnicas de geoprocessamento, ou seja, a tabulação cruzada no Spring (Instituto Nacional de Pesquisas Espaciais, 1999), o que possibilitou obter os valores de erro e acerto entre os dois métodos estudados.

\section{Fase 8: avaliação de dados espectrais na quantificação como auxílio no mapeamento visto na fase 6}

Com o objetivo de avaliar a possibilidade de atingir o terceiro nível categórico (grande grupo) da classificação de solos, foram realizadas análises estatísticas de predição de atributos do solo, conforme descrito a seguir. Na predição, valores dos atributos físicos - teores de areia, silte e argila - e químicos como MO, soma de bases (S), CTC, saturação por bases (V\%) e saturação por alumínio (m\%), utilizou-se a análise de regressão linear múltipla no programa Statistical Analysis System (SAS Institute, 1999). Inicialmente foi realizada a seleção das variáveis independentes - bandas e alturas - a serem consideradas no ajuste do modelo de regressão, por meio da análise passo a passo, para cada atributo do solo. Em seguida, realizou-se o teste de multicolinearidade para eliminação de uma ou mais variáveis que atuavam concomitantemente na estimativa de um mesmo atributo. A seqüência das análises é mostrada na Figura 1.

\section{Resultados e Discussão}

\section{Comportamento espectral dos solos}

Quatro classes de solos foram encontradas na área pelos métodos convencional e espectral, sendo a de maior expressão o Latossolo Amarelo Ácrico típico $\left(\mathrm{LAw}_{1}\right)$, originada de material argiloso e de baixa fertilidade natural. Por causa das aplicações de calcário e fertilizantes realizadas anteriormente para o cultivo de grãos, a saturação por bases na superfície é elevada, assim como os teores de P e K (Tabela 1).

A presença de material laterítico, em posição intermediária no relevo recoberto pelo material de origem da unidade $\mathrm{LAw}_{1}$, desenvolve-se o Latossolo Amarelo Ácrico plíntico $\left(\mathrm{LAw}_{2}\right)$, semelhante ao anterior tanto quanto a granulometria como no aspecto químico. Nas partes mais baixas da área desenvolve-se o Gleissolo Háplico Tb distrófico (GXbd), originado do mesmo material das unidades anteriores. Uma classe de menor expressão foi designada como Cambissolo Háplico alumínico típico (CXa).

A unidade $\mathrm{LAw}_{1}$ apresenta intensidade de reflectância das camadas B e $\mathrm{C}$ variando de 0,25 a 0,34 na faixa do espectro entre 700 e $2.150 \mathrm{~nm}$ por causa do seu caráter argiloso e teor de óxidos de ferro, conforme pode ser observado pelas nítidas feições entre 400-550 nm e 800-900 nm (Formaggio et al., 1996; Demattê et al., 2001). A camada A manteve-se em um patamar inferior aos 0,30 de reflectância ao longo de todo o espectro por causa dos maiores teores de MO que mascaram o efeito dos óxidos de ferro (Demattê \& Garcia, 1999) (Figura 2).

$\mathrm{Na}$ unidade $\mathrm{LAw}_{2}$ a camada superficial apresentou reflectância mais alta (Figura 2), possivelmente pelo fato de a MO neste solo ser constituída de material menos decomposto, tendo como causa a condição de menor 


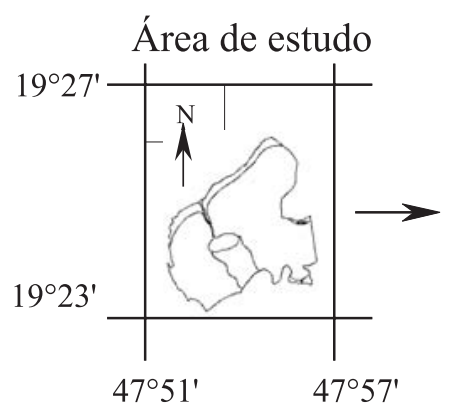

Demarcação dos

pontos para

tradagem

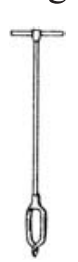

Georeferenciamento dos pontos

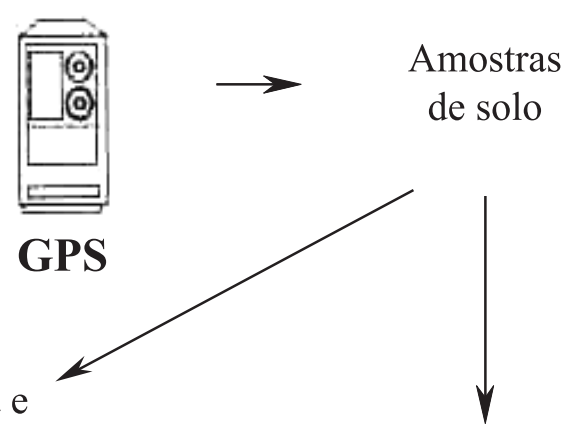

Aquisição dos dados espectrais

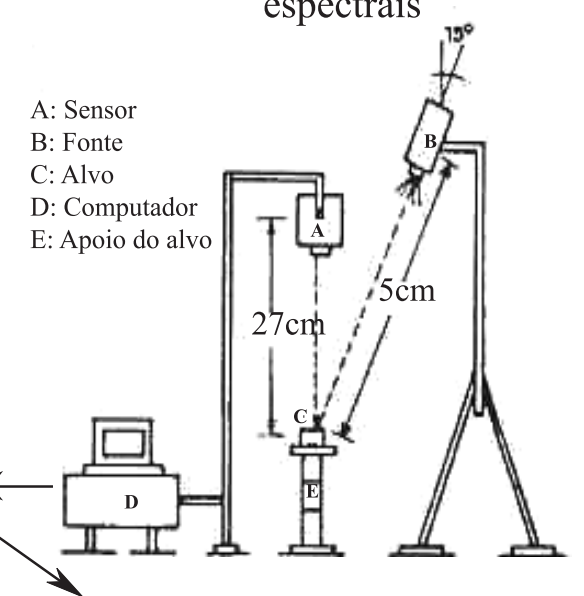

Mapa de Solos 1 Método convencional (Verdade de campo)

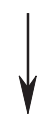

Tabulação cruzada (Spring)

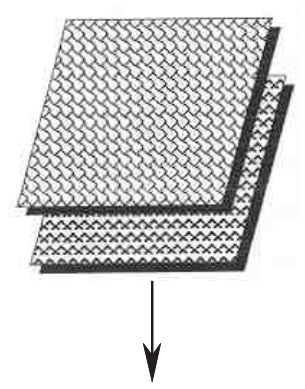

Interpretação da eficiência dos métodos

$$
1
$$

Mapa de Solos 2 por radiomeria
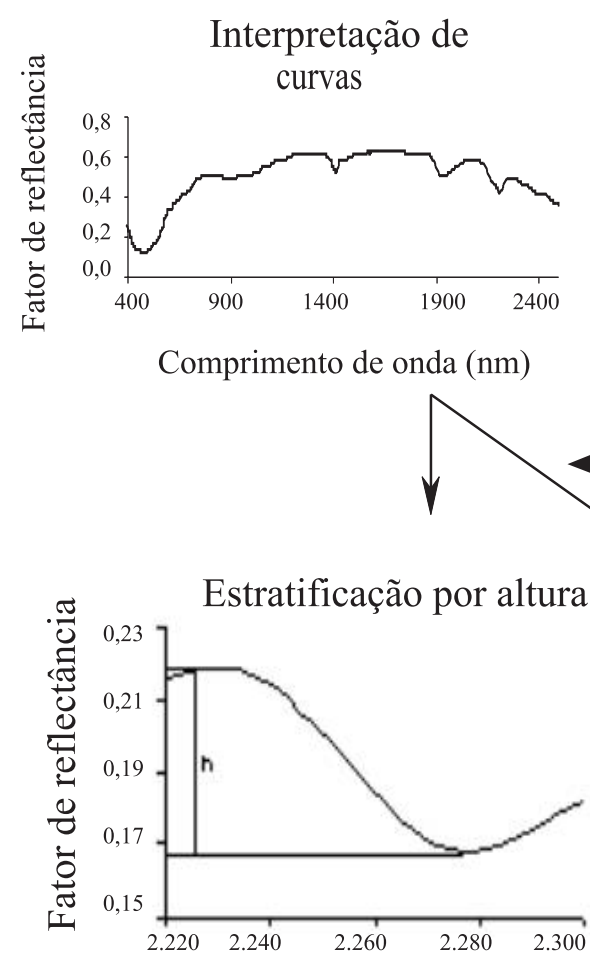

Estratificação por banda

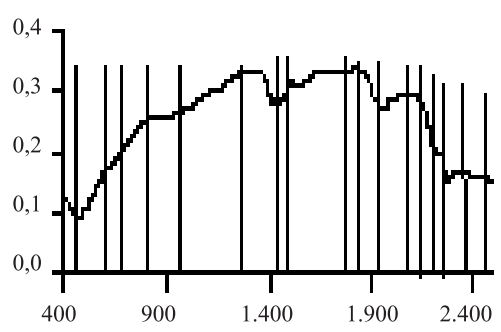

Comprimento de onda (nm)

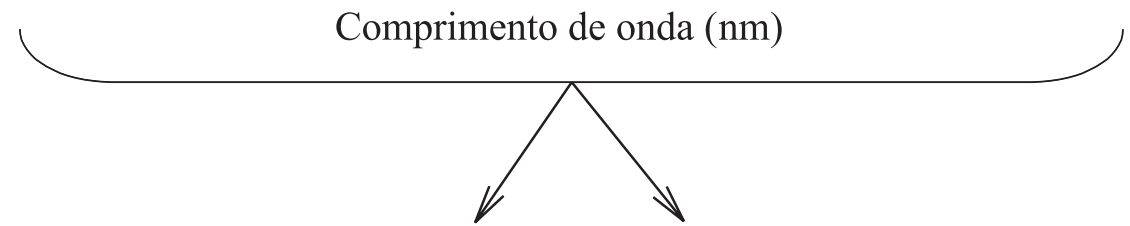

Analise de cluster + plani-altimetria + derivadas + análise Modelos de quantificação (passo-a-passo) qualitativa das curvas

Figura 1. Seqüência dos procedimentos utilizados. 
drenagem pela presença do material laterítico, o que reduz a taxa de decomposição da matéria orgânica.

Foram observadas feições de absorção em $1.400 \mathrm{~nm}$ e em $1.900 \mathrm{~nm}$, ocasionadas pela vibração das moléculas de água intercalar na estrutura cristalina dos minerais de argila, e em $2.200 \mathrm{~nm}$, devido à caulinita (Figura 2), corroborando os dados de Clemente et al. (2000) quando este autor estuda a mineralogia de solos derivados de diabásio. Tais bandas são menos intensas se comparadas às dos $\mathrm{CXa}$ e do GXbd, por causa dos maiores teores de minerais 2:1 desses solos. A concavidade em $650 \mathrm{~nm}$ constitui indicação da presença de óxidos de ferro no solo (Vitorello \& Galvão, 1996), assim como a banda em $2.265 \mathrm{~nm}$ ratifica a presença de gibbsita (Madeira Netto, 1996).

A resposta espectral da unidade GXbd permitiu inferir uma forte presença de MO na camada superficial (Figura 2), o que pode ser comprovado pela grande diferença de intensidade de reflectância, quando comparada com as camadas $\mathrm{B}$ e $\mathrm{C}$ ao longo de todo o espectro. Na faixa de 400 a $1.200 \mathrm{~nm}$ o formato das curvas nas três profundidades é côncava-convexa-côncava, indicando que embora haja altos teores de MO, os óxidos de ferro estão presentes. As bandas da água estão presentes em 1.400 e $1.900 \mathrm{~nm}$ e a banda de absorção em $2.200 \mathrm{~nm}$ indica a existência de mineralogia 1:1. É observada ainda a feição da gibbsita em $2.265 \mathrm{~nm}$ (Figura 2).

A unidade CXa apresenta pouca profundidade, textura muito argilosa em todo o perfil (Tabela 1) e oscilações da intensidade de reflectância entre as amostras das camadas A, B e C na faixa principal de atuação da MO (400-1.200 nm) (Figura 2).

Os óxidos de ferro, hematita e goethita, ocorrem nas três camadas do CXa, tendo em vista a mesma concavidade entre 400 e $550 \mathrm{~nm}$ (Demattê \& Garcia, 1999). Em relação às bandas da água intercalar (1.400 e $1.900 \mathrm{~nm}$ ), essas são mais intensas neste solo quando comparadas com os latossolos. A feição de absorção dos minerais de argila em $2.200 \mathrm{~nm}$ pode ser visualizada, mas com pouca nitidez, entretanto, a derivada segunda mostra em $2.200 \mathrm{~nm}$ a feição dupla da caulinita e a gibbsita entre $2.250 \mathrm{~nm}$ e $2.300 \mathrm{~nm}$, o que permite inferir que a mineralogia desse solo é $2: 1+1: 1$. O efeito da MO na camada A, na redução da sua reflectância e na atenuação da banda de absorção dos óxidos de ferro em 850-900 nm é também observado nesse solo.

\section{Comparação entre o mapa espectral e o mapa con- vencional}

Os mapas de solos obtidos pelo método convencional e pelo método espectral, no nível detalhado apresentam quatro classes de solos: $\mathrm{LAw}_{1}, \mathrm{LAw}_{2}$, GXbd e CXa (Figura 3). A comparação dos mapas foi realizada mediante tabulação cruzada entre as informações obtidas e expressa os valores de áreas coincidentes para cada classe de solo assim como as divergências.

Desta forma, o $\mathrm{LAw}_{1}$, que no mapa convencional ocupa 915,01 ha ou $72,24 \%$ da área (Tabela 2), no mapa espectral ocorre em 876,63 ha ou $69,21 \%$ da área e a área restante desta classe de solo, 38,38 ha, foi classifi-

Tabela 1. Valores médios dos atributos de solos em três profundidades ${ }^{(1)}$.

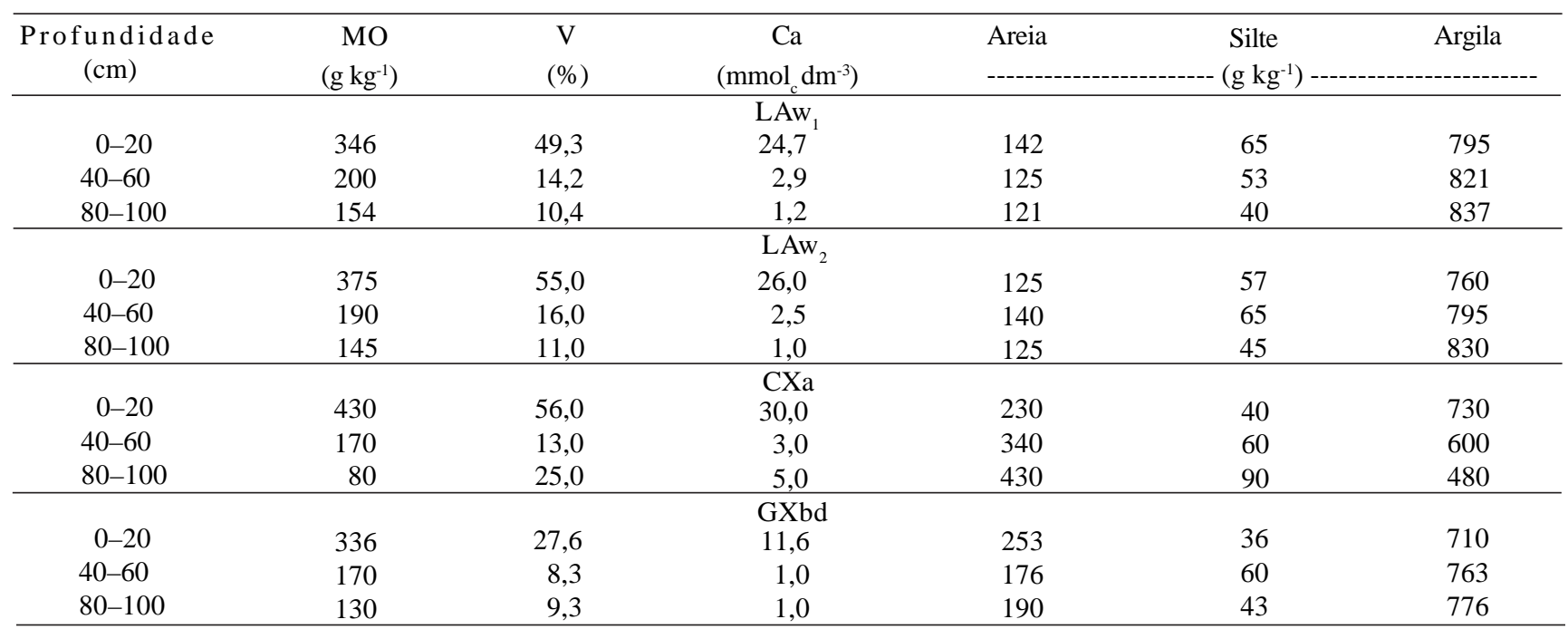

(1)MO: matéria orgânica; V: saturação por bases; LAw 1 : Latossolo Amarelo ácrico típico; LAw : Latossolo Amarelo ácrico plíntico; CXa: Cambissolo Háplico alumínico típico; GXbd: Gleissolo Háplico Tb distrófico. 

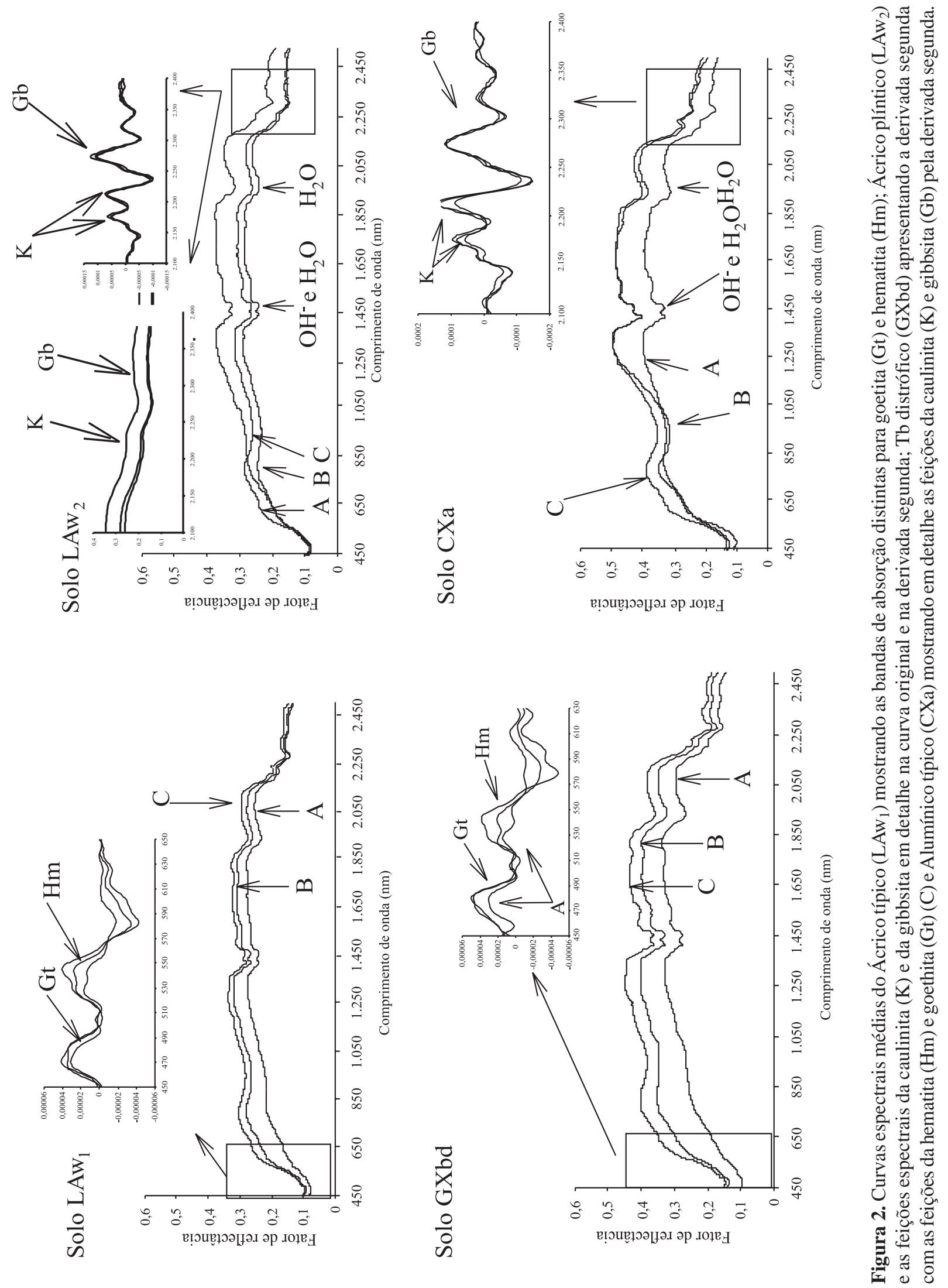

Pesq. agropec. bras., Brasília, v.39, n.12, p.1219-1229, dez. 2004 
cada no mapa espectral como $\mathrm{LAw}_{2}$ em 13,37 ha e como GXbd em 25,01 ha, assim como o $\mathrm{LAw}_{2}$ confundiu-se apenas com $\mathrm{LAw}_{1}$ e o GXbd com os dois LAw.

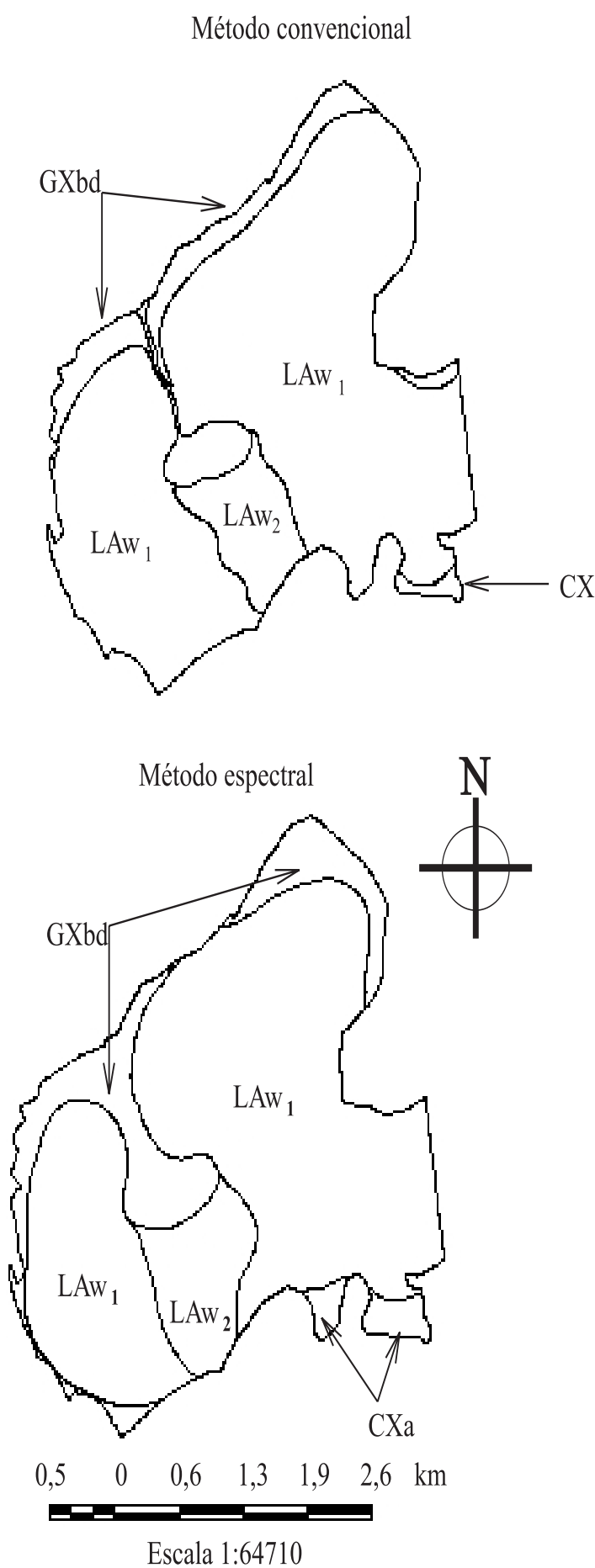

Figura 3. Mapas de solos obtidos pelos métodos convencional e espectral. $\mathrm{LAw}_{1}$ : Ácrico típico; $\mathrm{LAw}_{2}$ : Ácrico plíntico; CXa: Alumínico típico; GXbd: Tb distrófico.
O CXa apresentou uma área maior no mapa espectral, visto que ocupou uma parte da área representada pela unidade $\mathrm{LAw}_{1}$ no mapa convencional (Figura 3). A maior parte das confusões ocorreu na zona de transição entre as diferentes classes de solo. Por causa da complexidade destes locais, os dados espectrais não identificaram corretamente a classe de solo, além disso, como o cambissolo e o gleissolo ocupam uma área pequena proporcionalmente aos latossolos, qualquer ponto classificado erroneamente representa uma expressiva perda ou ganho de área.

Demattê et al. (2001), trabalhando em uma área de Mogi-Mirim, SP, mapearam cinco classes de solos (LVdf,

Tabela 2. Tabulação cruzada entre o mapa de solo espectral e o convencional, apresentando os valores absolutos (ha) e relativos $(\%)$.

\begin{tabular}{|c|c|c|}
\hline \multirow{2}{*}{$\begin{array}{l}\text { Mapa de solo } \\
\text { convencional }^{(2)}\end{array}$} & \multicolumn{2}{|c|}{ Mapa de solo radiométrico ${ }^{(1)}$} \\
\hline & (ha) & $(\%)$ \\
\hline & \multicolumn{2}{|c|}{$\mathrm{LAw}_{1}^{(3)}$} \\
\hline $\mathrm{LAw}_{1}$ & 876,63 & 69,21 \\
\hline $\mathrm{LAw}_{2}$ & 39,82 & 3,14 \\
\hline $\mathrm{CXa}^{2}$ & 22,38 & 1,77 \\
\hline GXbd & 93,42 & 7,38 \\
\hline \multirow[t]{2}{*}{ Total } & $1.032,25$ & 81,5 \\
\hline & \multicolumn{2}{|c|}{$\mathrm{LAw}_{2}^{(4)}$} \\
\hline $\mathrm{LAw}_{1}$ & 13,37 & 1,06 \\
\hline $\mathrm{LAw}_{2}$ & 73,27 & 5,78 \\
\hline $\mathrm{CXa}^{2}$ & 0,00 & 0,00 \\
\hline GXbd & 3,21 & 0,25 \\
\hline \multirow[t]{2}{*}{ Total } & 89,85 & 7,09 \\
\hline & \multicolumn{2}{|c|}{$\mathrm{CXa}^{(5)}$} \\
\hline $\mathrm{LAw}_{1}$ & 0,00 & 0,00 \\
\hline $\mathrm{LAw}_{2}$ & 0,00 & 0,00 \\
\hline $\mathrm{CXa}^{2}$ & 8,93 & 0,71 \\
\hline GXbd & 0,00 & 0,00 \\
\hline \multirow[t]{2}{*}{ Total } & 8,93 & 0,71 \\
\hline & \multicolumn{2}{|c|}{$\mathrm{CXbd}^{(6)}$} \\
\hline $\mathrm{LAw}_{1}$ & 25,01 & 1,97 \\
\hline $\mathrm{LAw}_{2}$ & 0,01 & 0,00 \\
\hline $\mathrm{CXa}$ & 0,00 & 0,00 \\
\hline GXbd & 110,55 & 8,73 \\
\hline \multirow[t]{2}{*}{ Total } & 135,56 & 10,70 \\
\hline & \multicolumn{2}{|c|}{ Total } \\
\hline $\mathrm{LAw}_{1}$ & 915,01 & 72,24 \\
\hline $\mathrm{LAw}_{2}$ & 113,01 & 8,92 \\
\hline $\mathrm{CXa}^{2}$ & 31,31 & 2,48 \\
\hline GXbd & 207,18 & 16,36 \\
\hline Total & $1.266,59$ & 100,00 \\
\hline \multicolumn{3}{|c|}{$\begin{array}{l}\text { (1) Mapa de solos gerado mediante análise quantitativa e qualidade de } \\
\text { dados radiométricos obtidos em laboratório. }{ }^{(2)} \text { Mapa de solos gerado } \\
\text { pelo método convencional. }{ }^{(3)} \text { Latossolo Amarelo ácrico típico. } \\
\text { (4) Latossolo Amarelo ácrico plíntico. }{ }^{(5)} \text { Cambissolo Háplico alumínico } \\
\text { típico. }{ }^{(6)} \text { Gleissolo Háplico Tb distrófico. }\end{array}$} \\
\hline
\end{tabular}


LVd, PVAd1, GX+RU e CXbe) e constataram boa correlação entre o mapa espectral e o mapa convencional detalhado demonstrando que os dados espectrais obtidos em laboratório são úteis no levantamento de solos.

\section{Quantificação de atributos do solo}

Equações de regressão múltipla foram ajustadas na predição dos valores de oito atributos do solo a partir de sua resposta espectral. Com exceção do silte e da satu- ração por alumínio, os demais atributos do solo apresentaram coeficientes de determinação $\left(\mathrm{R}^{2}\right)$ acima de $70 \%$ (Tabela 3). Esses resultados corroboram os obtidos por Janik et al. (1998), Nanni (2000) e Fiorio (2002). Altos coeficientes dos atributos físicos como areia e argila têm sido alcançados, visto que estes parâmetros influenciam de forma mais intensa a resposta espectral do solo.

A soma de bases (S), a capacidade de troca catiônica (CTC) e a saturação por bases (V\%) apresentaram

Tabela 3. Equações de regressão múltipla para estimativa de atributos do solo utilizando-se as 22 bandas e 13 alturas obtidas da estratificação das curvas espectrais das amostras de solo.

\begin{tabular}{cc}
\hline Equação de regressão múltipla ${ }^{(1)}$ & $\mathrm{R}^{(2)}$ \\
\hline Areia & $0,8272 *$ \\
$139,54997-35,64517 \mathrm{~B} 12+6768,79550 \mathrm{~B} 16-5913,97533 \mathrm{~h} 9+5761,36763 \mathrm{~h} 8-1028,72642 \mathrm{~B} 11-$ & \\
$7161,33287 \mathrm{~B} 9-11613 \mathrm{~B} 4-6687,20075 \mathrm{~B} 8+6226,25615 \mathrm{~B} 3+1936,08113 \mathrm{~h} 2+13690 \mathrm{~B} 5-13538 \mathrm{~B} 6-$ & \\
$4155,76327 \mathrm{~B} 19+225,20268 \mathrm{~B} 21-9626,29353 \mathrm{~B} 17-10777 \mathrm{~h} 13-2385,81244 \mathrm{~B} 22+9304,17112 \mathrm{~B} 10+$ & \\
$2836,20199 \mathrm{~h} 7-920,78920 \mathrm{~B} 1+1795,47810 \mathrm{~h} 6-6166,00904 \mathrm{~h} 5-5227,56035 \mathrm{~h} 12+4580,64718 \mathrm{~B} 18-$ & \\
$8028,13974 \mathrm{~h} 10+1344,20463 \mathrm{~B} 13+5082,89416 \mathrm{~B} 20+11469 \mathrm{~B} 7$ &
\end{tabular}

Silte

$80,55259+816,76425 \mathrm{~h} 1+5146,13060 \mathrm{~h} 8-2443,58316 \mathrm{~B} 3+1767,92086 \mathrm{~B} 4-1241,80482 \mathrm{~h} 2+17623 \mathrm{~B} 16$

$0,4726^{*}$ - 1164,91025B12 - 4719,96946B13 - 11367h9 - 11120B15 - 2484,51559B17 + 1705,95224B11 $1017,55182 \mathrm{~B} 10-393,77653 \mathrm{~h} 4+1580,80458 \mathrm{~B} 22$

\section{Argila}

$806,17525-327,59768 \mathrm{~B} 22+3063,68258 \mathrm{~B} 9-6453,41683 \mathrm{~B} 11+7043,91579 \mathrm{~B} 4+4306,08301 \mathrm{~B} 12+$ $5557,40740 \mathrm{~B} 1+554,79958 \mathrm{~B} 8+2238,64037 \mathrm{~B} 7-6573,67936 \mathrm{~h} 8+11920 \mathrm{~h} 13-5246,12838 \mathrm{~B} 21+$ $3349,79867 \mathrm{~B} 19-1491,95398 \mathrm{~h} 6+3204,57962 \mathrm{~B} 17+504,19224 \mathrm{~B} 10-952,58743 \mathrm{~B} 15-2207,59866 \mathrm{~h} 9-$ $34468 \mathrm{~B} 16+34688 \mathrm{~B} 18-1756,30074 \mathrm{~h} 7+41537 \mathrm{~h} 10+4500,59291 \mathrm{~h} 1-6661,18759 \mathrm{~h} 2+1705,47505 \mathrm{~B} 5-$ 16186B3 - 4470,06051B 13 - 3677,45055h12

\section{Matéria orgânica}

$25,90501-126,17752 \mathrm{~h} 11+888,15012 \mathrm{~h} 13-1051,17547 \mathrm{~B} 5+119,33747 \mathrm{~h} 9-518,36861 \mathrm{~B} 16+75,33500 \mathrm{~B} 12$ $-606,20426 \mathrm{~h} 4-70,79898 \mathrm{~h} 3-59,32577 \mathrm{~B} 1+448,03185 \mathrm{~B} 4+1430,22346 \mathrm{~B} 8-41,23356 \mathrm{~B} 6-137,67664 \mathrm{~B} 22$

$0,9206^{*}$ $-660,06668 \mathrm{~B} 10+73,00525 \mathrm{~h} 6+507,54336 \mathrm{~B} 17$

Soma de bases

$35,32975+328,32698 \mathrm{~B} 1-1530,24315 \mathrm{~B} 2+290,35017 \mathrm{~B} 3-3795,58920 \mathrm{~h} 10+782,28693 \mathrm{~B} 7+43,33704 \mathrm{~B} 6$ - 2482,07627B $17+1867,33097 \mathrm{~h} 7-521,59743 \mathrm{~B} 20+4627,76345 \mathrm{~B} 16-3885,09207 \mathrm{~B} 18+1829,04997 \mathrm{~B} 19$ $+148,93604 \mathrm{~h} 6-3309,44531 \mathrm{~B} 11-1070,55364 \mathrm{~h} 2+3660,48480 \mathrm{~B} 10$

Capacidade de troca catiônica

$52,52688-1384,77232 \mathrm{~B} 1+1970,87937 \mathrm{~B} 2-1053,18014 \mathrm{~B} 22+962,62017 \mathrm{~B} 7+847,72852 \mathrm{~B} 15-$

$0,8463^{*}$ $240,83478 \mathrm{~h} 8+346,42511 \mathrm{~h} 7-13,50904 \mathrm{~B} 12+2274,28969 \mathrm{~h} 13-190,97467 \mathrm{~B} 3-386,34389 \mathrm{~B} 5+$ $1595,44804 \mathrm{~B} 9-648,10002 \mathrm{~B} 10-1541,01734 \mathrm{~h} 5-1697,85160 \mathrm{~B} 11+725,87451 \mathrm{~h} 4-65,03891 \mathrm{~h} 3$

\section{Saturação por bases}

$60,78000+723,09372 \mathrm{~B} 1+68,45647 \mathrm{~h} 10+1242,46713 \mathrm{~h} 12+223,66061 \mathrm{~B} 4+738,43224 \mathrm{~B} 6-2033,51560 \mathrm{~B} 2$ $-1505,39767 \mathrm{~h} 2+1458,23938 \mathrm{~h} 13+77,19616 \mathrm{~h} 7-228,37898 \mathrm{~h} 9-142,63240 \mathrm{~h} 6+501,78371 \mathrm{~h} 11$

\section{Saturação por alumínio}

20,67293 - 11359h10 - 466,36771B4 - 7474,58739h12 + 1239,15545B14 - 990,94949B13 - 3227,68534h8 $-4024,41363 \mathrm{~B} 17+10200 \mathrm{~B} 16-6423,74035 \mathrm{~B} 9+2404,36977 \mathrm{~B} 8-1891,76124 \mathrm{~h} 5-5733,50554 \mathrm{~B} 18+$ 3781,55030B5 - 3505,18050B1 - 3251,94180h1 - 4824,50206B19 - 5919,43257B22 + 2308,71535h9 $3079,72051 \mathrm{~h} 2-2200,44497 \mathrm{~h} 13+3058,70611 \mathrm{~B} 12+7323,70914 \mathrm{~B} 20+3860,53727 \mathrm{~B} 10$

${ }^{(1)} \mathrm{B} 1 \ldots \mathrm{B} 22, \mathrm{~h} 1 \ldots$... 13 : bandas e alturas selecionadas, respectivamente. ${ }^{*}$ Significativo a $5 \%$ de probabilidade pelo teste $\mathrm{F}$. 
valores de $\mathrm{R}^{2}$ elevados concordando com Dunn et al. (2002), porém tais atributos normalmente mostram coeficientes com valores menores que 50\% (Demattê \& Garcia, 1999; Fiorio, 2002). Poucos trabalhos tem sido realizados para explicar a influência das propriedades químicas na resposta espectral dos solos devido à natureza dinâmica das reações do solo.

Os valores obtidos em relação à matéria orgânica também são muito variáveis. Assim, Ben-Dor \& Banin (1995) e Demattê \& Garcia (1999) apresentaram coeficientes de $50 \%$ e $45 \%$, respectivamente, enquanto Demattê et al. (2001) obtiveram $\mathrm{R}^{2}$ de $79 \%$ e Coleman et al. (1991) de 68\%, menos significativos do que os obtidos neste trabalho (92\%) (Tabela 3).

A utilização destes modelos pode permitir alcançar até o terceiro nível categórico na classificação. Entretanto, por causa do número de amostras utilizadas e da presença de apenas quatro classes de solos, os modelos desenvolvidos não podem ser extrapolados para outras regiões, sendo necessário estudos específicos relacionados a este tópico.

A aplicação dos modelos desenvolvidos (Tabela 3) pode ser feita obtendo-se os dados espectrais de amostras de terra com valores de V\% desconhecidos, que seriam introduzidos na equação e o resultado do V\% estimado seria utilizado para auxiliar na determinação do terceiro nível categórico na classificação desta amostra.

\section{Conclusões}

1. A análise do comportamento espectral dos solos permite determinar atributos como a granulometria, mineralogia, matéria orgânica e o ferro que, aliados à curva espectral em todo o espectro eletromagnético estudado, auxiliando na classificação do solo, até o segundo nível categórico.

2. O sensoriamento remoto espectral, as curvas de nível e o trabalho de campo permitem realizar a delimitação de solos detalhadamente.

3. A quantificação da areia, argila, CTC, V\%, S, m\% e da matéria orgânica por dados espectrais indica a possibilidade de atingir o terceiro nível categórico na classificação de solo.

\section{Referências}

ANDRONIKOV, V.L.; DOBROLVSHIY, G.V. Theory and methods for the use of remote sensing in the study of soils. Mapping Science and Remote Sensing, v.28, p.92-101, 1991.

BEN-DOR, E. Quantitative remote sensing of soil properties. Advances in Agronomy, v.75, p.173-243, 2002.

BEN-DOR, E.; BANIN, A. Near-infrared analysis as a simultaneously method to evaluate spectral featureless constituents in soils. Soil Science, v.159, p.259-269, 1995.

BEN-DOR, E.; BANIN, A. Near-infrared reflectance analysis of carbonate concentration in soils. Applied Spectroscopy, v.44, p.1064-1069, 1990.

CAMARGO, A.O. de; MONIZ, A.C.; JORGE, J.A.; VALADARES, J.M. Métodos de análise química, mineralógica e física de solos. Campinas: Instituto Agronômico, 1986. 94p. (IAC. Boletim Técnico, 106).

Clemente, C.A.; DEMATtê, J.A.M.; MAFRA, A.L.; BENTIVENHA, S.R.P. Reflectância espectral e mineralogia de materiais formados sobre diabásio. Scientia Agricola, v.57, p.159$168,2000$.

COLEMAN, T.L.; AGBU, P.A.; MONTGOMERY, O.L. Spectral differentiation of soils and soil properties: is it possible from space platforms? Soil Science, v.155, p.283-293, 1993.

COLEMAN, T.L.; AGBU, P.A.; MONTGOMERY, O.L.; GAO, T.; PRASAD, S. Spectral band selection for quantifying selected properties in highly weathered soils. Soil Science, v.151, p.355361, 1991.

DEMATTÊ, J.A.M.; GARCIA, G.J. Alteration of soil properties through a weathering sequence as evaluated by spectral reflectance. Soil Science Society of America Journal, v.63, p.327-342, 1999.

DEMATTÊ, J.A.M.; DEMATTÊ, J.L.I.; CAMARGO, W.P.; FIORIO, P.R.; NANNI, M.R. Remote sensing in the recognition and mapping of tropical soils developed on topographic sequences. Mapping Science and Remote Sensing, v.38, p.79-102, 2001.

DUNN, B.W.; BEECHER, H.G.; BATTEN, G.D.; CIAVARELLA, $S$. The potential of near-infrared reflectance spectroscopy for soil analysis - a case study from the Riverine Plain of south-eastern Australia. Australian Journal of Experimental Agriculture, v.42, p.607-614, 2002.

EMBRAPA. Centro Nacional de Pesquisa de Solos (Rio de Janeiro, RJ). Sistema brasileiro de classificação de solos. Brasília: Embrapa-SPI/Embrapa-CNPS, 1999. 412p.

EPIPHANIO, J.C.N.; FORMAGGIO, A.R.; VALERIANO, M.M.; OLIVEIRA, J.B. Comportamento espectral de solos do Estado de São Paulo. São José dos Campos, Instituto Nacional de Pesquisas Espaciais, 1992. 131p. Relatório INPE.

FIORIO, P.R. Dados radiométricos obtidos nos níveis terrestre e orbital na avaliação de solos. 2002. 198. Tese (Doutorado). Escola Superior de Agricultura Luiz de Queiroz, Piracicaba.

FORMAGGIO, A.R.; EPIPHANIO, J.C.N.; VALERIANO, M.M. Comportamento espectral $(450-2.450 \mathrm{~nm})$ de solos tropicais de São Paulo. Revista Brasileira de Ciência do Solo, v.20, p.467474, 1996. 
GALVÃO, L.S.; VITORELLO, I.; FORMAGGIO, A.R. Relationship of spectral reflectance and color among surface and subsurface horizons of tropical soil profiles. Remote Sensing of Environment, v.61, p.24-33, 1997.

GEOPHYSICAL ENVIRONMENTAL RESEARCH CORP, GER. Mark V dual field of view IRIS manual. Version 1.3. Milbook: New York, 1996. 63p.

INSTITUTO NACIONAL DE PESQUISAS ESPACIAIS (São José dos Campos, SP). Software Spring: versão 3.3. São José dos Campos, 1999 (Manual do usuário on line).

JANIK, L.J.; MERRY, R.H.; SKJEMSTAD, J.O. Can mid infrared diffuse reflectance analysis replace soil extractions? Australian Journal of Experimental Agriculture, v.38, p.681-696, 1998.

LEMOS, R.C. de; SANTOS, R.D. dos. Manual de descrição e coleta de solo no campo. 3.ed. Campinas: Sociedade Brasileira de Ciência do Solo, $1996.84 \mathrm{p}$.

MADEIRA NETTO, J.S. Spectral reflectance properties of soils. Photo Interpretation, v.34, p.59-70, 1996.

McBRATNEY, A.B.; MENDONÇA SANTOS, M.L.; MINASNY, B. On digital soil mapping. Geoderma, v.117, p.3-52, 2003.
NANNI, M.R. Dados radiométricos obtidos em laboratório e no nível orbital na caracterização e mapeamento de solos. 2000. 366p. Tese (Doutorado) - Escola Superior de Agricultura Luiz de Queiroz, Piracicaba.

NICODEMUS, F.E.; RICHMOND, J.C.; HSIA, J.J.; GINSBERG, I.W.; LIMPERIS, T. Geometrical considerations and nomenclature for reflectance. U.S. Department of Commerce, 1977. 52p. (NBS Monograph, 160).

RAIJ, B. van.; QUAGGIO, J.A.; CANTARELLA, H.; FERREIRA, M.E.; LOPES, A.S.; BATAGLIA, C.O. Análise química do solo para fins de fertilidade. Campinas: Fundação Cargill, 1987. 170p.

SAS INSTITUTE (Cary, Estados Unidos). SAS, software: user's guide: version 8.2. Cary, 1999. 219p.

SAVITZKY, A.; GOLAY, M.J.E. Smoothing and differentiation of data by simplified least squares procedures. Analytical Chemistry, v.36, p.1627-1639, 1964.

VITORELLO, I.; GALVÃO, L.S. Spectral properties of geologic materials in the 400 to $2.500 \mathrm{~nm}$ range: Review of applications to mineral exploration and lithologic mapping. Photo Interpretation, v.34, p.77-99, 1996.

Recebido em 4 de setembro de 2003 e aprovado em 24 de setembro de 2004 